\title{
Slip and Wear at a Corner with Coulomb Friction and an Interfacial Strength
}

\author{
Zupan $\mathrm{Hu}^{\dagger}$, Wei $\mathrm{Lu}^{\dagger, *}$ and M. D. Thouless ${ }^{\dagger, t, *}$ \\ ${ }^{\dagger}$ Department of Mechanical Engineering \\ ${ }^{\ddagger}$ Department of Materials Science \& Engineering \\ University of Michigan, Ann Arbor, MI 48109, USA
}

\begin{abstract}
Traditional analyses of slip at corners of contacts, based on linear elasticity and a Coulomb friction law, are very sensitive to the details of local geometries, owing to the effects of elastic singularities. Following the use of cohesive-zone models to address such issues in mode-II fracture, we present analyses of slip and wear at corners of contacts when a finite interfacial shear strength is incorporated with a Coulomb friction law. We show that the concept of an instantaneous cohesive-length scale, borrowed from the field of fracture mechanics, can be used to describe the nature of stress fields around corners, and defines when linear-elasticity and Coulomb friction can provide an accurate description of the interfacial behavior. We also show that the sensitivity of slip analyses to geometrical details decreases when the cohesive-length scale increases. We also show that the cohesive strength of an interface plays a crucial role in the propagation of a wear scar across an interface. If only Coulomb slip is assumed to occur, a wear scar may not progress beyond the original stick-slip boundary. If a finite interfacial shear strength is introduced into the analysis, the wear scar can propagate along the interface.
\end{abstract}

Keywords: Cohesive zone; Cohesive-length scale; Coulomb friction; Shear strength; Wear; Partial slip;

May 27, 2015

*Corresponding authors: telephone: +1 7347635289 (MDT); +1 7346477858 (WL);

fax: +1 734647 3170; e-mail: thouless@umich.edu; weilu@umich.edu 


\section{Introduction}

The life of many engineering components can be limited by fretting wear [1] induced by cyclic slip between two contacting surfaces [2]. This wear is often initiated at the corners of contacts, where there are high stresses that can be singular in elastic analyses. However, an assumption of Coulomb friction in these analyses leads to a prediction of slip and wear only if the coefficient of friction, $\mu$ is low enough, with the critical value of $\mu$ depending on the details of the corner geometry. Similar issues of sensitivity to local geometry are inherent for problems of crack propagation in interfacial fracture mechanics. In that field, cohesive-zone models, which incorporate the concept of a finite interfacial strength, have been found to be useful tools to resolve some of the unrealistic complexities associated with singular stresses, while retaining the general features of fracture mechanics that make it useful at larger scales $[3,4,5]$. In this paper we apply the insight provided by the field of interfacial fracture mechanics to interfacial slip, showing that the assumption of a finite shear-strength changes the slip and wear behavior at corners in important ways.

The contact across an interface between two bodies can be described by three regimes of behavior that depend on the geometry and the loads [6]. The first regime is full-stick, where the interface is effectively bonded across its entire length, and the two bodies act as a single entity. The second regime is full-slip, where there is relative motion between the two bodies along the entire interface. The third regime is partial-slip, where the two materials slide relative to each other along some parts of the interface, and are effectively bonded along others. Wear is associated with the energy dissipated by sliding [6,7], and the analysis of wear requires modeling the relative slip along the interface between the two bodies. The full-stick and full-slip 
regimes can be relatively easy to describe; partial-stick can provide more of a challenge. However, this last phenomenon is an important aspect of wear at the corners of contacts and, therefore, forms the focus of this study.

Coulomb's law is a common criterion used to determine when slip occurs. This law states that the magnitude of the interfacial shear stress, $q$, is limited by the product of the local applied pressure across the interface, $p$, and the coefficient of friction, $\mu$ :

$$
|q| \leq \mu p
$$

Slip occurs if this condition cannot be satisfied without allowing a relative shear displacement across the interface. The coefficient of friction is generally assumed to be a constant that is characteristic of the interface; if it is assumed to vary, non-linear effects are introduced [8].

Coulomb's law allows for the possibility of an arbitrarily high interfacial shear stress, if the local pressure is high enough. Indeed, Coulomb slip at a corner results in singular shear stresses. This unphysical result can be avoided by assuming that the magnitude of the shear stresses is limited by an interfacial shear strength, $\hat{\tau}$, that is independent of local pressure $[9,10]$. For example, the shear strength of the contacting materials could provide an upper bound to this parameter. Local equilibrium then requires a second condition that

$$
|q| \leq \hat{\tau} .
$$

This concept of a single-valued interfacial shear strength is commonly used in fibercomposite models $[11,12]$, as well as in thin-film and composite-laminate cracking problems $[3,13]$. 
The stresses along an interface near a corner are generally singular for elastic bodies in contact. The strength of the singularity depends on the details of the geometry, and is the same for both the shear stress and the pressure $[14,15,16]$. This means that the ratio of the shear to normal stress is constant near the corner, no matter how high the stresses are. So, depending on the magnitude of the friction coefficient, either slip by the Coulomb criterion occurs everywhere within the singular region, or there is complete sticking within the singular region. Partial slip occurs when the Coulomb condition is met within the singular field, but not outside it. However, the stress field associated with Coulomb slip is still singular, both the contact pressure and the shear stress increase without limit as the corner is approached.

The purpose of this paper is to examine how the assumption of a limiting value for the interfacial shear stress, as given by Eq. (2), affects slip at the corner of contacts, and how this might influence the evolution of wear. In the next section we give a brief summary of how the interfacial stresses near corners depend on geometry and slip conditions. We then show how these stress distributions and the slip are affected by the assumption of a finite interface strength. This is followed by a demonstration of how the sensitivity of slip to details of the corner geometry is reduced by invoking a finite interfacial strength. The final part of the paper demonstrates how the assumption of a finite interface strength can have a significant effect on the evolution of a wear scar, allowing it to propagate across an interface, rather than arresting at the initial slip-stick boundary. 


\section{Background}

\subsection{No slip}

In this paper, we consider two elastic bodies with the same properties, contacting each other along flat surfaces. Figure 1 shows a magnified view near the corner of the contact for such a system. If the interface does not satisfy either of the two slip conditions of Eqns. (1) and (2), the stresses are given by the elastic solution for a wedge. Close to the corner, the stresses are singular. For example, along a line that bisects the corner (Fig. 1), the singular components of the normal and shear stresses are given by $[17,18]$

$$
\begin{gathered}
p_{\theta \theta}(r)=K_{I} r^{\lambda_{I}-1} \\
q_{r \theta}(r)=K_{I I} r^{\lambda_{I I}-1},
\end{gathered}
$$

where $r$ is the distance from the corner, $K_{I}$ and $K_{I I}$ are the mode-I (symmetrical) and mode-II (anti-symmetrical) stress-intensity factors, and the strengths of the singularities, $\lambda_{I}$ and $\lambda_{I I}$, depend on the exterior angle, $\phi$. The two stress-intensity factors depend on the detailed geometry of the corner, but they also depend on the macroscopic geometry, and on the applied loads. They are analogous to those used in fracture mechanics, and describe the effects of the geometry and loads ${ }^{1}$.

Equation (3) describes the singular stresses along a line that bisects the exterior angle. Of particular interest are the normal pressure, $p$, and shear stress, $q$, along the

\footnotetext{
${ }^{1}$ As can be seen from the definition of the singular stress field, the stress-intensity factors usually used in the fracture mechanics literature differ from those used in the friction literature by a factor of $\sqrt{2 \pi}$.
} 
Figure 1: Geometry of a corner with an exterior angle $\phi$, and an interface along which sliding can occur

interface [18]:

$$
\begin{aligned}
& p(x)=f_{1} K_{I} x^{\lambda_{I}-1}+f_{2} K_{I I} x^{\lambda_{I I}-1} \\
& q(x)=f_{1} g_{1} K_{I} x^{\lambda_{I}-1}+f_{2} g_{2} K_{I I} x^{\lambda_{I I}-1}
\end{aligned}
$$

where $x$ is the distance along the interface (Fig. 1), and $f_{1}, f_{2}, g_{1}$ and $g_{2}$ are functions of $\phi$. The geometry we will generally consider in this paper is one for which $\phi=90^{\circ}$. For this case, the stresses along the interface are given by [18]

$$
\begin{aligned}
& p(x)=0.7303 K_{I} x^{-0.4555}-1.0873 K_{I I} x^{-0.0915} \\
& q(x)=0.3966 K_{I} x^{-0.4555}+0.2381 K_{I I} x^{-0.0915}
\end{aligned}
$$




\subsection{Coulomb slip}

A comparison between Eqs. (4) and (1) reveals that slip will always occur in the singular region at the corner of a contact if $\mu<g_{1}(\phi)$, since $\lambda_{I}$ dominates the stress field close to the corner. In particular, Eq. 5 shows that slip will occur if $\mu<0.543$ for the right-angled geometry considered in this paper. If the Coulomb slip condition is met within the singular region, the asymptotic stress field develops a different singularity, $\lambda_{s}$, that is a function of both $\phi$ and $\mu$. The interfacial stresses close to the corner are then given by [18]

$$
\begin{gathered}
p(x)=K_{s} x^{\lambda_{s}-1}, \\
q(x)=\mu K_{s} x^{\lambda_{s}-1},
\end{gathered}
$$

where $K_{s}$ is a stress-intensity factor that depends on the geometry and loads. For a right-angled corner, $\lambda_{s}$ is given by the solution to $[19,20]$

$$
\left[\sin ^{2}\left(\pi \lambda_{s} / 2\right)-\lambda_{s}^{2}\right] \cos \left(\pi \lambda_{s}\right)+(1 / 2) \sin ^{2}\left(\pi \lambda_{s}\right)+\mu \lambda_{s}\left(1+\lambda_{s}\right) \sin \left(\pi \lambda_{s}\right)=0 .
$$

Coulomb slip does not resolve the issue of singular stresses. Theoretically, even if slip occurs, the shear stresses are infinite at the corners. Obviously, in practice, the stresses are limited by a finite strength of the interface. The question addressed in this paper, is the extent to which the assumption of a finite interface strength affects the slip and wear conditions at the corner of a contact. This is examined by adapting the concept of cohesive-zone models for interfacial fracture, where the introduction of finite strengths allows smooth transitions between regimes in which singular elastic fields dominate (albeit, limited by the finite strengths), and regimes where these fields provide very poor descriptions of the interfacial mechanics. 


\section{$2.3 \quad$ Cohesive zones}

Linear-elastic fracture mechanics is predicated on an assumption that the only interfacial property controlling fracture is the interfacial toughness. Cohesive-zone models incorporate a cohesive strength into the description of the interface. Mode-II cohesive laws provide an analogue for sliding problems without adhesive bonding. For a homogenous system in plane stress, a nominal mode-II fracture length can be defined as $[5,21,22]$

$$
\zeta_{I I}=E \Gamma_{I I} / \hat{\tau},
$$

where $E$ is the modulus, and $\Gamma_{I I}$ is the mode-II toughness of an interface. If the nominal fracture length is small compared to any appropriate geometrical length, then the toughness controls crack propagation, and the interfacial stresses follow the elastic stress field close enough to the crack tip for the singularity to be experienced. If the nominal fracture length is large compared to any appropriate geometrical length, then the cohesive strength controls crack propagation, and the interfacial stresses are essentially uniform along much of the interface.

If one assumes there is no fracture associated with slip, the concept of a nominal fracture length is not useful, because $\Gamma_{I I}$ isn't defined. However, the concept of a fracture length has been generalized to the notion of an instantaneous cohesive length, which has meaning at any point during the loading of an interface $[5,3]$. For mode-II cracks, this instantaneous cohesive length is given by

$$
\xi_{I I}=\bar{E}^{*} \delta_{s}^{2} / \mathcal{W}_{I I_{o}}
$$

where $\bar{E}^{*}$ is the effective modulus of two materials contacting across an interface (which is $E$ for the plane-stress, homogeneous case considered here ${ }^{2}$ ), $\delta_{s}$ is the slip

\footnotetext{
${ }^{2} \bar{E}=E$ in plane stress and $E /\left(1-\nu^{2}\right)$ in plane strain, where $\nu$ is Poisson's ratio. In the fracture
} 
distance at the crack tip (defined as the point where the shear tractions go to zero), and $\mathcal{W}_{I I_{o}}$ is the work done in sliding (per unit area) by the shear tractions at the crack tip.

It appears that this definition of an instantaneous cohesive length should be useful for slip problems, since there is no reference to fracture. In particular, if a cohesive slip displacement of magnitude $\delta_{s}$ occurs at a critical shear stress of $\hat{\tau}$, the instantaneous cohesive length is given by

$$
\xi_{I I}=\bar{E}^{*} \delta_{s} / \hat{\tau}
$$

The instantaneous cohesive length can be compared to the smallest relevant dimension of the system to give a cohesive-length scale. By analogy to fracture mechanics, the magnitude of this cohesive-length scale is expected to determine whether slip can be considered to be small-scale (and controlled by elasticity), or not. If the ratio is significantly smaller than unity, it is expected that a portion of the elastic stress field will exhibit the singularity appropriate to the geometry of the corner. If the cohesive-length scale is larger, it is expected that the stresses will be constant over a relatively large portion of the interface. These concepts will be illustrated in the results that follow. ${ }^{3}$

\footnotetext{
literature, it makes sense to define $\bar{E}^{*}=2 \bar{E}_{1} \bar{E}_{2} /\left(\bar{E}_{1}+\bar{E}_{2}\right)$, where the subscripts refer to the materials on either side of an interface, so $\bar{E}$ is recovered in the homogeneous case.

${ }^{3}$ We simplify the problem in this paper by neglecting any physical limit on the normal pressure. Therefore, the solutions that follow have singular normal stresses. These are not expected to influence the slip behavior, but could be accommodated by an appropriate mode-I cohesive law for the interface. We also ignore the fact that imaginary components of the stress field can be introduced by elastic mismatch across an interface [14]. These complications can be resolved by invoking a cohesive-zone model for the interface [4]. Furthermore, when $\xi_{I I}$ is sufficiently small, it needs to be compared to microstructural or interfacial asperity length scales, not geometrical scales [3].
} 


\section{Numerical model}

The results in this paper were generated using a finite-element model to calculate the stresses and slip along an interface near a sharp corner. The geometry and mesh used is shown in Fig. 2. It was a plane-stress model consisting of a rectangular block of height, $h$, and length, $l$, in contact with a large substrate with the same elastic modulus $E$. The macroscopic exterior angle between the block and substrate was always $90^{\circ}$. Attention was focussed on the left-hand corner of this geometry (the portion of the interface in which the two objects always remain in contact). We used an aspect ratio of $l / h=20$, and confirmed that this was large enough for the solutions that are presented to be unaffected by the right-hand boundary. We also confirmed that the substrate was deep enough for the effects of the bottom boundary (which was constrained in all directions) to be negligible within a reasonable level of numerical error. The absolute values of the parameters were varied to explore the utility of the non-dimensional groups used. In the results that are presented, only the non-dimensional groups that affect the results are reported.

The block was subjected to a uniform distributed normal pressure, $p_{\infty}$, and a uniform distributed tangential stress, $q_{\infty}$. The normal pressure was applied first as a pre-load, and the tangential stress was then applied to explore the subsequent slip. The coefficient of friction $\mu$ was varied to explore the stick and slip regimes predicted by Coulomb's law. The cohesive strength of the interface, $\hat{\tau}$, was given an infinite value when exploring Coulomb friction, and a finite value when exploring the effect of a cohesive strength. Only conditions in which there was no global slip were considered; no-slip equilibrium conditions were always satisfied from a macroscopic perspective. In all cases, the contacting bodies were perfectly elastic, so the only non-linear effects 


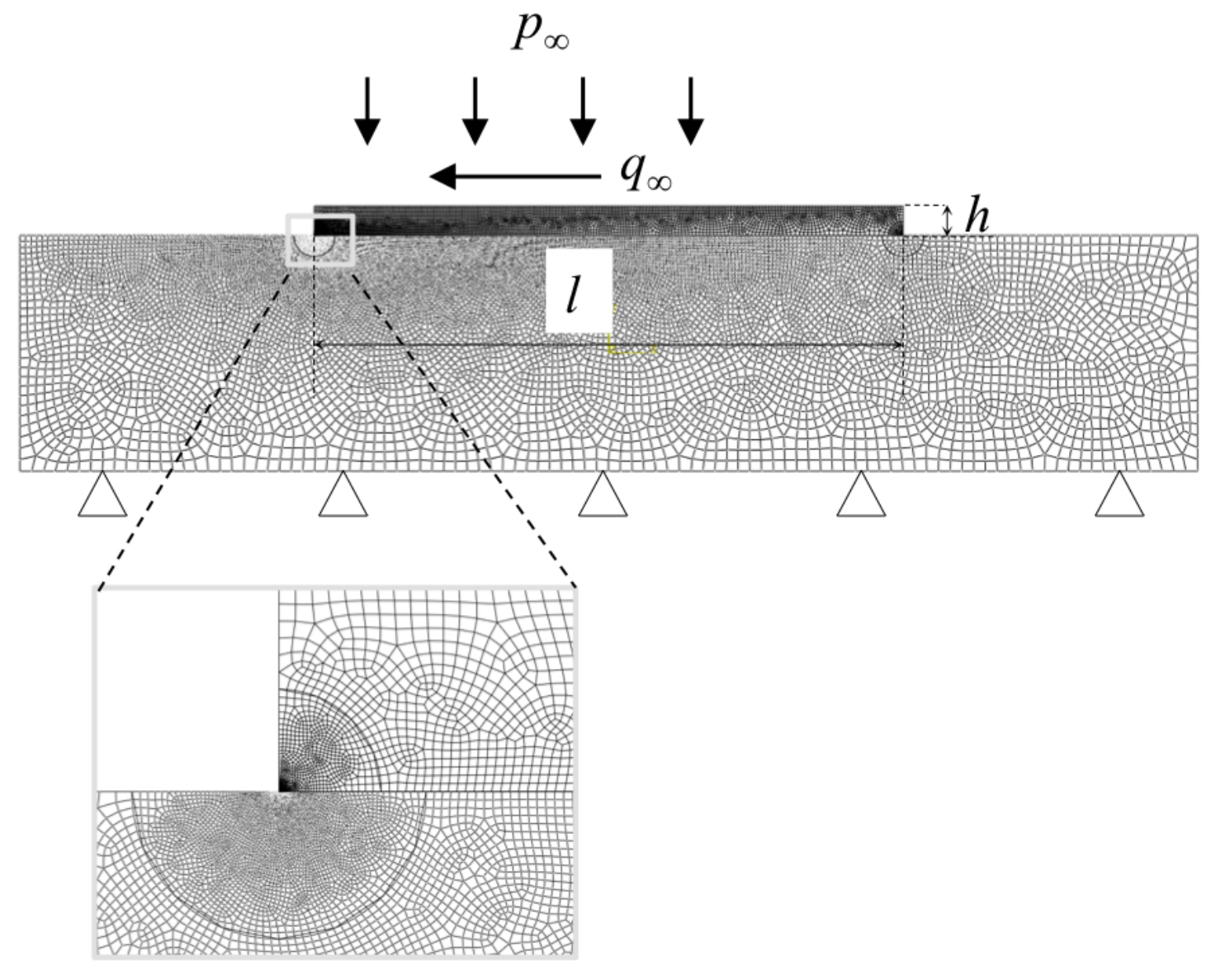

Figure 2: Finite-element mesh of the geometry studied in this paper. 
were associated with slip at the interface.

The simulations were performed using the commercial finite-element package ABAQUS. Four-node, bilinear, coupled temperature-displacement elements (CPE4T) were used. Standard options available in the package were used for the contact elements [23]. A master-slave contact was defined along the contact interface; in every time increment, the slave nodes were adjusted so that there was no penetration between the contact surfaces. The contact was formulated according to the "surface-to-surface" option. The particular method for the discretization was set to the "surface-to-surface" option, in which the contact conditions are enforced over neighboring nodes. The slip conditions for Coulomb friction and a finite shear strength were also set within the usual options available in the program. However, since very small amounts of relative motion across an interface can evolve from the finite-element calculations, a separate sub-routine was used to compare the values of $q$ and $q / p$ to $\hat{\tau}$ and $\mu$ and to establish whether these displacements were associated with slip or numerical uncertainty. This was useful for determining slip-stick boundaries.

In order to capture the singular stress field correctly, we refined the mesh around the contact corners using a bias seeding. Typically, the mesh had more than 18,000 elements, with the smallest elements being refined to a value of $0.002 h$. Validation that the mesh was fine enough was achieved by plotting the stresses along the interface on a log-log plot, and comparing the slope of the line close to the interface with the expected dominant singularity. For example, the numerical solutions resulted in a predicted singularity of $\lambda_{I}=0.544 \pm 0.003$ for a non-slipping interface, and $\lambda_{s}=0.651 \pm 0.001$ for a slipping interface with $\mu=0.27$. These are in excellent agreement with the expected values of 0.5445 and 0.6503 , respectively, and 
confirms that the quality of our mesh and technique is acceptable to within a very reasonable level of uncertainty. Once it was confirmed that the strength of the singularity in the numerical solutions was correct, the values of the stress-intensity factors could also be found from the plots. For example, when $q_{\infty} / p_{\infty}=0.25$, these values $\operatorname{are} K_{I} h^{\lambda_{I}-1} / p_{\infty}=0.558 \pm 0.003$, and $K_{s} h^{\lambda_{s}-1} / p_{\infty}=0.796 \pm 0.001$ for $\mu=0.27$. Error bars indicating estimates of our numerical uncertainties have been included on all the plots.

\section{Results and discussion}

\subsection{Larger coefficients of friction}

The distributions of the shear and normal stresses along the interface are shown in Fig. 3 for a case in which $\mu>0.543$, so slip would not occur if only Coulomb friction acted. Since $l / h \gg 1$ in these calculations, the characteristic length scale used to normalize the parameters in these plots is $h$. The plot labelled $\xi_{I I} / h=0$ corresponds to the limiting case of an infinite shear strength. Since there is no slip, this stress field corresponds to the elastic solution for the fully-bonded case. In particular, the straight-line portion of this plot has a slope that is in excellent agreement with the expected singularity of $\lambda_{I}=0.5445$. The curves for the two non-zero values of $\xi_{I I} / h$ were calculated using different values of the applied stresses, keeping the values of both $q_{\infty} / p_{\infty}$ and $\xi_{I I} / h$ constant. $\xi_{I I} / h$ was kept constant by trail and error: using the values of $\delta_{s}$ computed for different values of $q_{\infty} / E$ to determine $\xi_{I I}$. All the stress plots were coincident for fixed values of both $q_{\infty} / p_{\infty}$ and $\xi_{I I} / h$. This indicates that the instantaneous cohesive-length scale provides a means of characterizing the slip. 
(a)

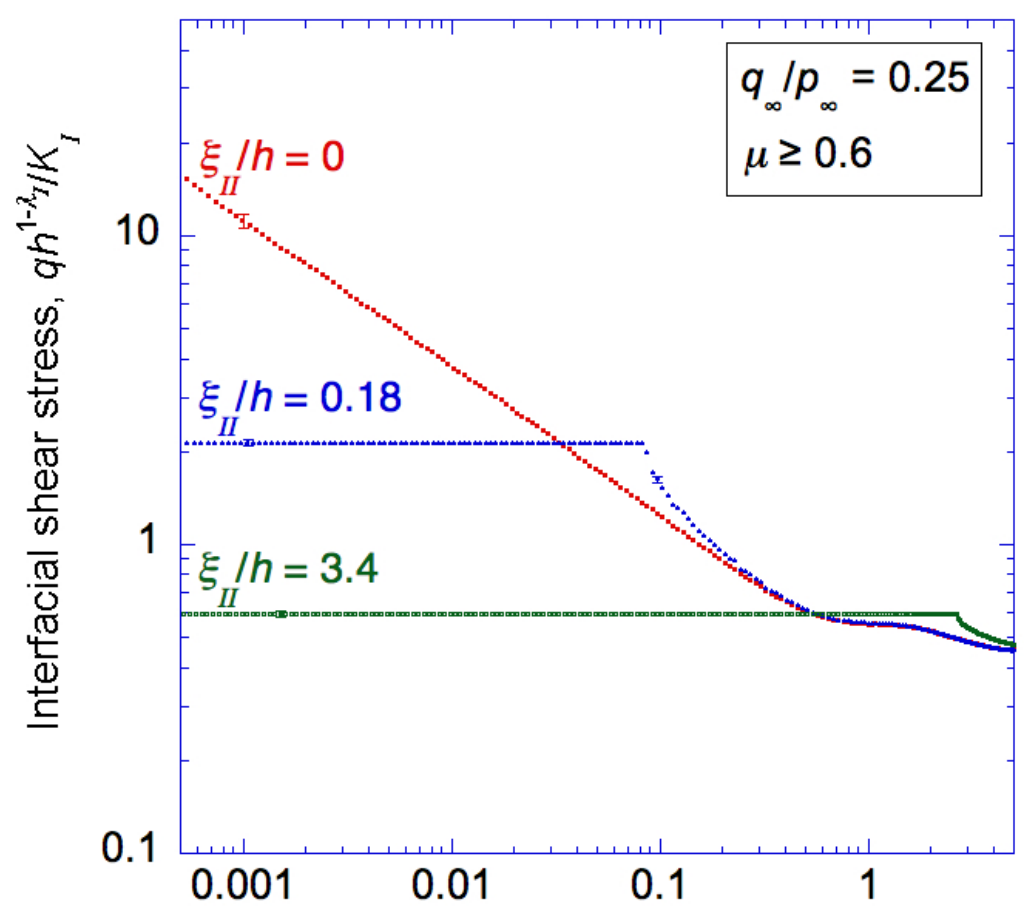

\section{Distance from corner, $x / h$}

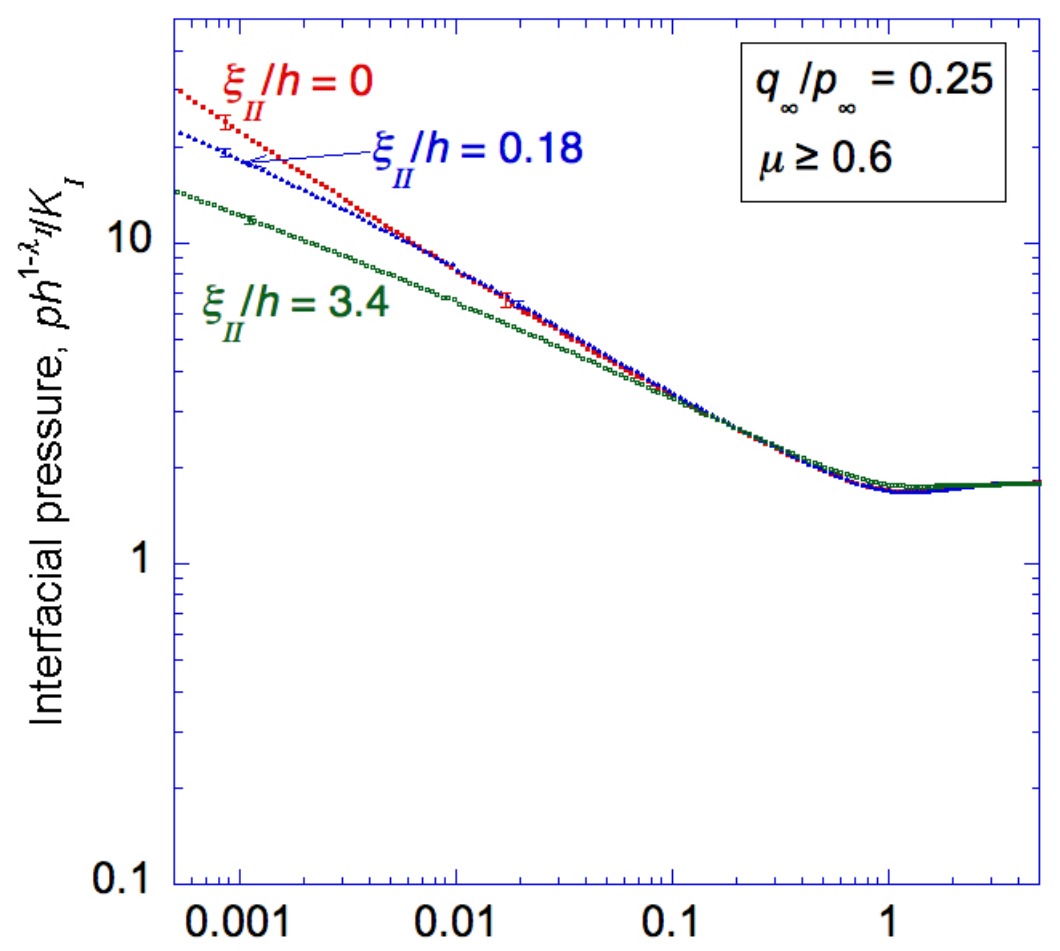

(b) Distance from corner, $x / h$ 
(c)

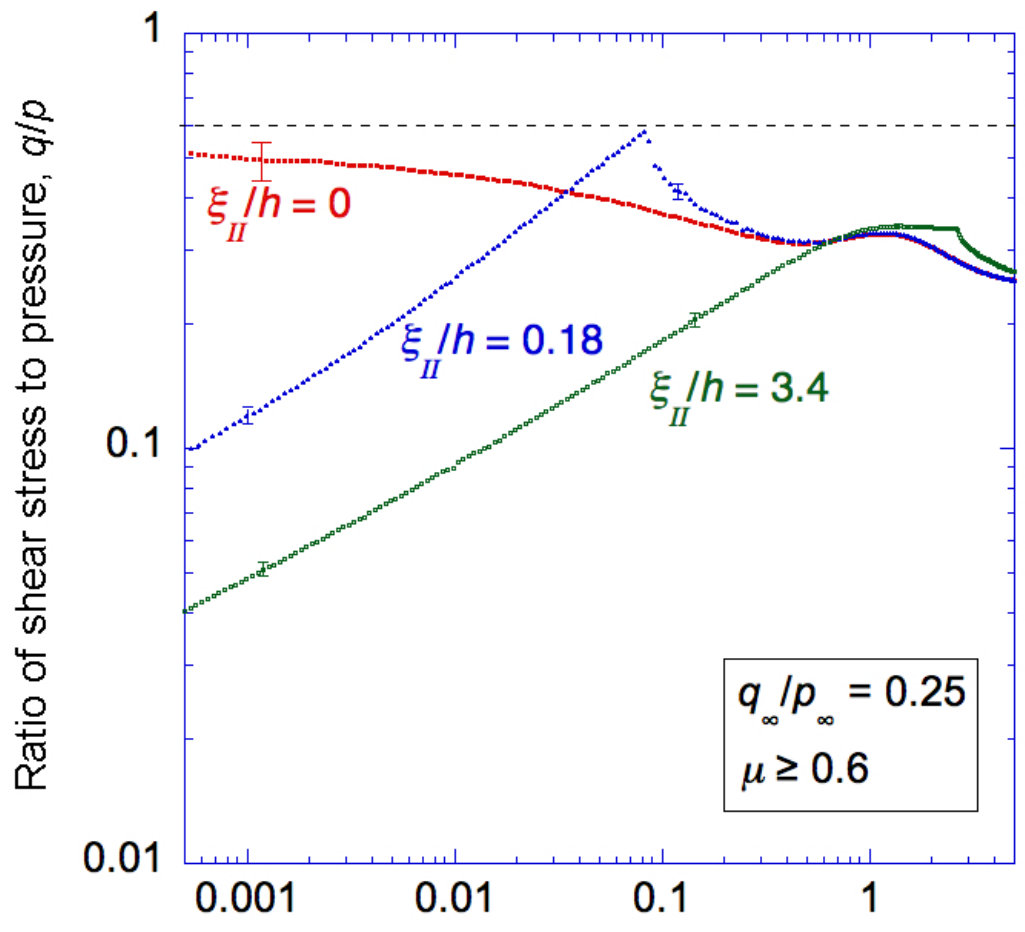

\section{Distance from corner, $x / h$}

Figure 3: Interface stress distributions with $\mu \geq 0.6$. Unless the interfacial shear strength is finite, no slip can occur since $\mu>0.543$. (a) Distribution of shear stresses along the interface for three representative cases: (i) no slip, with $\xi_{I I} / h=0$, (ii) small-scale slip, with $\xi_{I I} / h=0.18$, and (iii) large-scale slip, with $\xi_{I I} / h=3.4$. (b) Normal stress distributions along the interface for the same three values of $\xi_{I I} / h$. (c) The corresponding ratios between the shear and normal stresses. 
Figure 3 shows that when $\xi_{I I} / h$ is reasonably small, slip is embedded within the singular elastic field, so that the slip can be described as being small-scale. The instantaneous cohesive-length scale increases as the extent of slip increases, and large-scale slip occurs when it extends so far that there is no region where the stresses exhibit singular behavior. As expected, outside the slip region, the shear stresses follow the far-field elastic solution. This behavior is exactly what is expected by analogy to cohesive-zone models for cracks with similar instantaneous cohesive-length scales. While the shear stresses are limited by the interfacial strength near the corner, the normal pressures still show singular fields, even in the slip region (although the singularity is weaker than predicted by the no-slip condition). In practice, of course, these normal stresses will be limited by yield of the contacting materials.

Whether there is any regime of Coulomb-controlled slip or not can be seen by looking at the ratio of $q / p$, as plotted in Fig. 3(c). There is a peak in this ratio at the edge of the slip regime. If the value of $\mu$ is greater than the magnitude of this peak, then the strength-controlled slip regime ends in a region of sticking. If the value of $\mu$ is smaller than the magnitude of this peak, there will be two regions of slip: one controlled by the interfacial strength, the other controlled by Coulomb's law. This peak rises from 0.543 to a maximum of $0.74 \pm 0.02$, as $\xi_{I I} / h$ increases from zero to 0.005. The peak then falls with a further increase in the cohesive-length scale.

\subsection{Smaller coefficients of friction}

Figure 4 shows plots similar to those of Fig. 3, but with a much smaller friction coefficient of $\mu=0.27$. This is below the critical value of 0.543 for this geometry, so Coulomb slip can occur. In these plots the stresses have been normalized by the 
stresses for the singular field with slip, so they are of the form of $q / K_{s} h^{\lambda_{s}-1}$, where $\lambda_{s}=0.6503$ is the appropriate singularity for slip with $\mu=0.27$ (see Eq. (6)).

In this case, since there is both Coulomb slip and finite-strength slip, we need to distinguish between the two regions, so as to obtain the appropriate value of $\delta_{s}$ needed to evaluate $\xi_{I I} / h$ from Eq. (10). The regions where slip is controlled by $\hat{\tau}$ can be identified from the regions of constant shear stress in Fig. 4(a). The regimes of Coulomb slip can be identified from the regions where the ratio of $q / p=\mu$ in Fig. 4(c). Coulomb slip extended out to beyond the limits shown in Fig. 4(c), but this was always embedded in the elastic field corresponding to a stuck (or bonded) interface, with no influence from the other boundary.

There will always be a regime of strength-limited slip in the partial slip zone near the corner of a contact. However, as discussed above, Coulomb slip can also occur just outside this region before the fully-stuck zone is encountered. It is also possible for the strength-limited slip region to end at a stick region. These different types of behavior are illustrated in the map of Fig. 5, showing the effect of $\mu$ for one specific value of cohesive strength.

\subsection{Sensitivity to corner geometry}

One of the major effects of a cohesive zone in fracture is that it reduces the sensitivity to the details of any defects. Larger cohesive-length scales result in less sensitivity to singularities that arise in elastic analyses, and are associated with reduced notch sensitivity [22]. In this section, we examine how a cohesive zone might affect the sensitivity of slip predictions to the assumed details of a corner. This is done by 
(a)

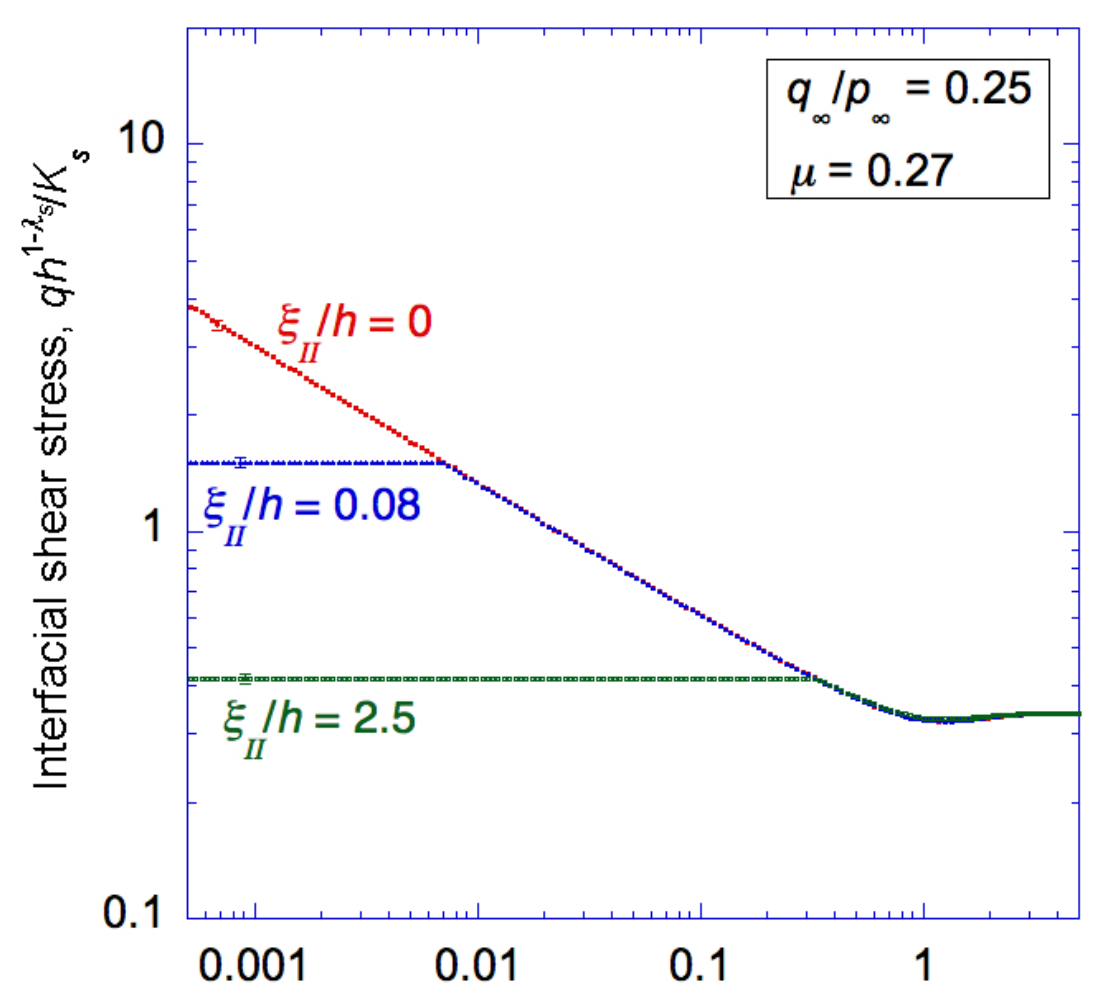

Distance from corner, $x / h$

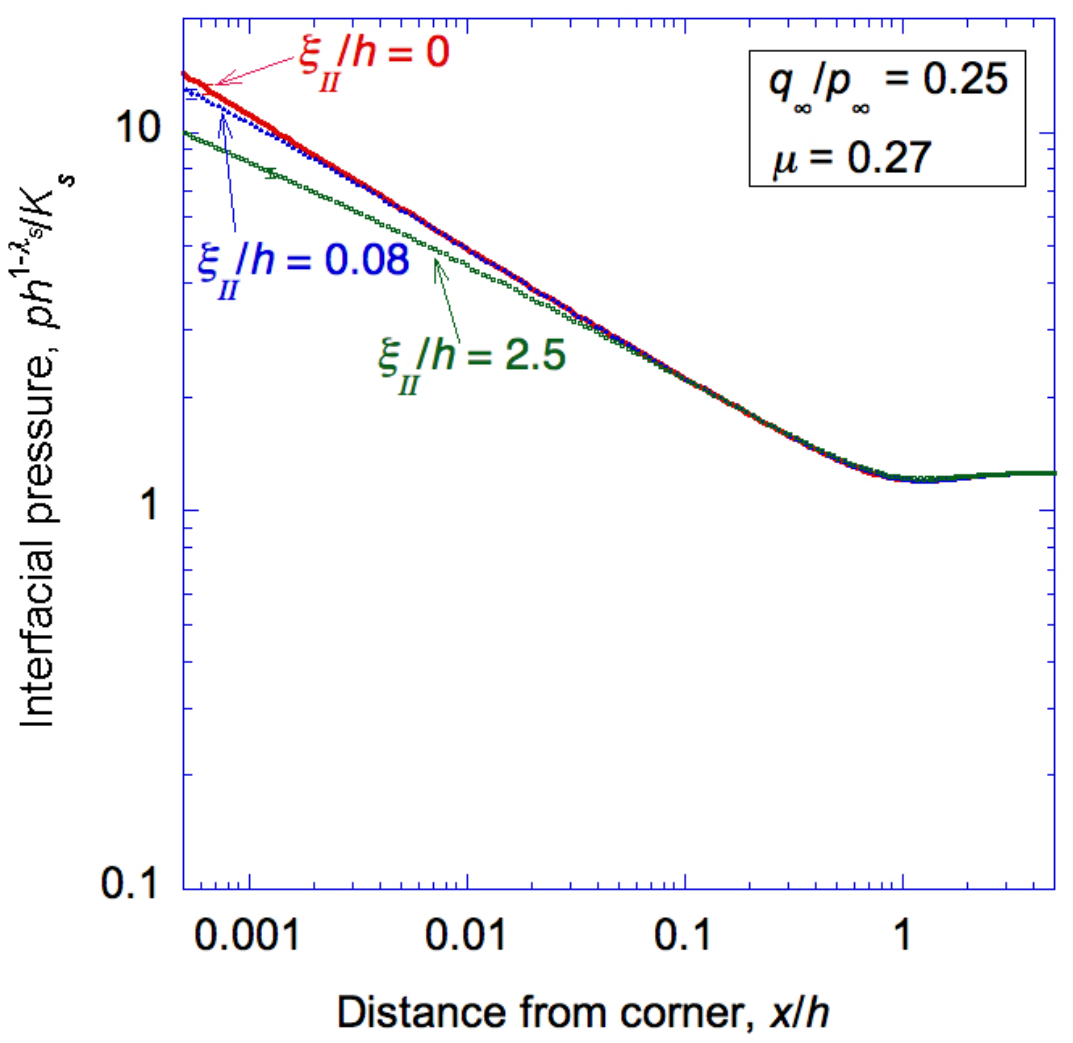

(b) 
(c)

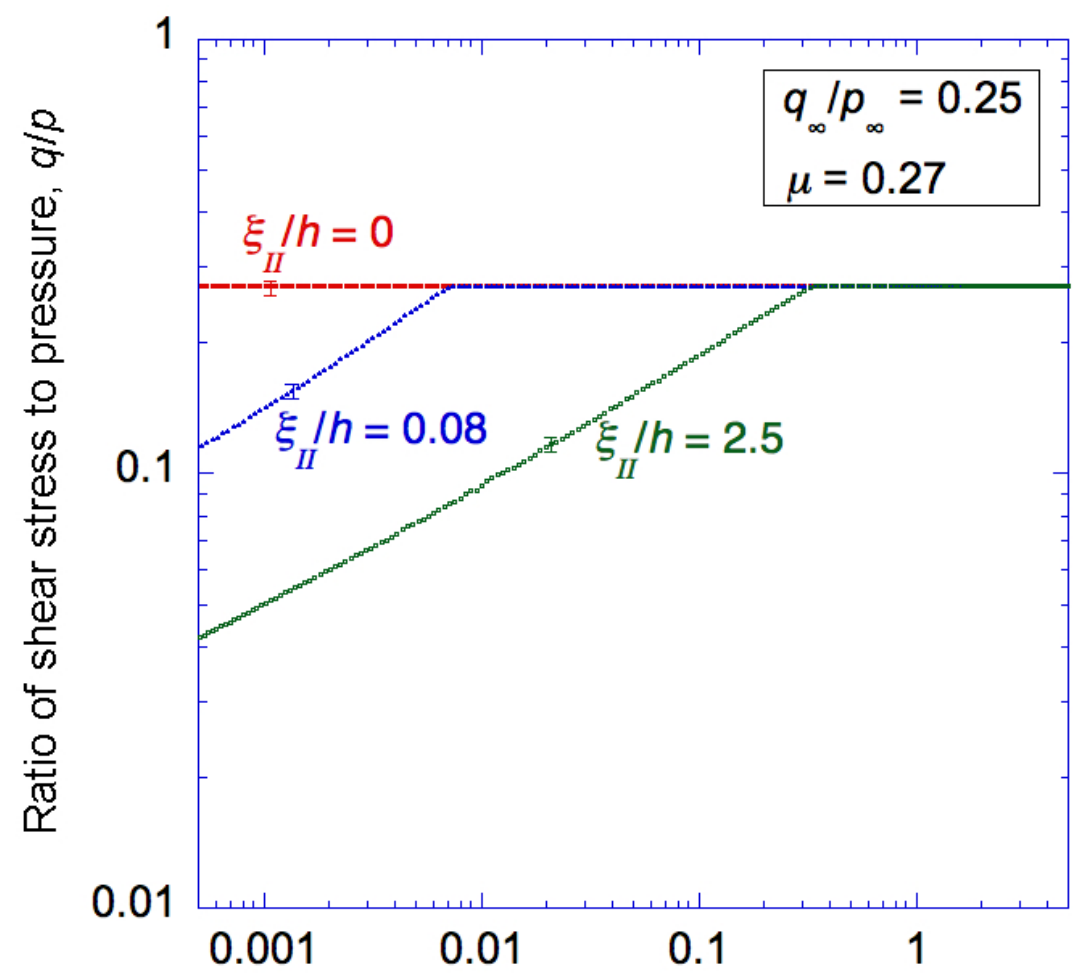

Figure 4: Interface stress distributions with $\mu=0.27$. The slip zone controlled by a finite interfacial shear strength is embedded in the Coulomb slip zone. (a) Distribution of shear stresses along the interface for three representative cases: (i) no slip $\left(\xi_{I I} / h=0\right),($ ii $)$ small-scale slip $\left(\xi_{I I} / h=0.08\right)$, and (iii) large-scale slip $\left(\xi_{I I} / h=\right.$ $2.5)$. (b) Normal stress distributions along the interface for the same three values of $\xi_{I I} / h$. (c) The corresponding ratios between the shear and normal stresses. 


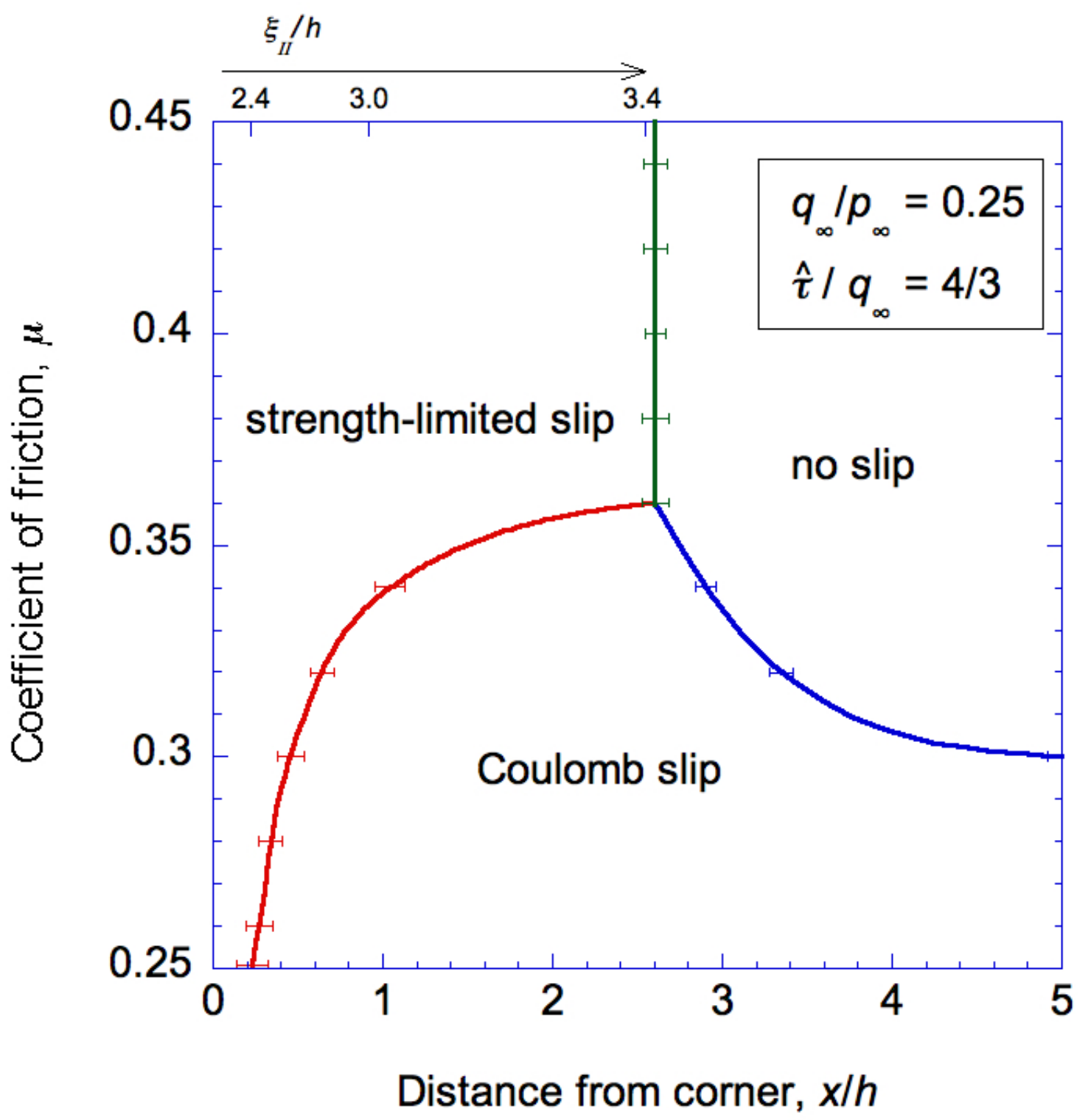

Figure 5: Slip-mechanism map showing how the slip mechanism depends on the coefficient of friction $\mu$ and the distance ahead of the corner, for the specific conditions of $q_{\infty} / p_{\infty}=0.25$. and $\hat{\tau} / q_{\infty}=4 / 3$. For these conditions, and partial slip, the corresponding values of $\xi_{I I} / h$ are in the range of about 2.4 to 3.4. Below $\mu=0.25$, there is complete slip along the interface. 
examining three different models, each with the same macroscopic geometry of a contact with a $90^{\circ}$ external angle (Fig. 6), but with different details for the corner at a smaller length scale. One of these models has a $90^{\circ}$ external angle to the smallest scale of the numerical model. Another of these geometries has a small notch, so that the actual angle of contact with the interface is $45^{\circ}$. The last of these geometries has a smooth contact with the interface, formed by the arc of a circle meeting the interface tangentially. The calculations are performed for a coefficient of friction given by $\mu=0.34$ for all three geometries.

The stresses for the three geometries are shown in Fig. 7 when there is an infinite shear strength, so slip is controlled by Coulomb friction only. The extent of slip can be seen most easily from Fig. $7(\mathrm{c})$, which shows the ratio of the shear to normal pressure along the interface. As can be seen in that figure, both the $90^{\circ}$ and smooth corner exhibit Coulomb slip near the corner, with the $90^{\circ}$ corner having a longer slip distance. The $45^{\circ}$ corner exhibits interesting behavior of being stuck at the corner, but with a region of internal slip. ${ }^{4}$

\footnotetext{
${ }^{4}$ The local singularity at a $45^{\circ}$ corner has a value of $\lambda_{I}=0.4950$, and there is complete stick at the edge if $\mu>0.2132$. The singular field associated with a small $45^{\circ}$ notch within a macroscopic $90^{\circ}$ corner, may be embedded within a larger-scale singular field corresponding to the macroscopic geometry, resulting in the possibility of either full stick or partial internal slip, depending on the value of $\mu$ and the size of the notch. For the geometry shown in Fig. 6(b), there would be no slip anywhere if $\mu$ is greater than about 0.38 , whereas the geometry of Fig. 6(a) would exhibit partial slip for this value of $\mu$.
} 
a)
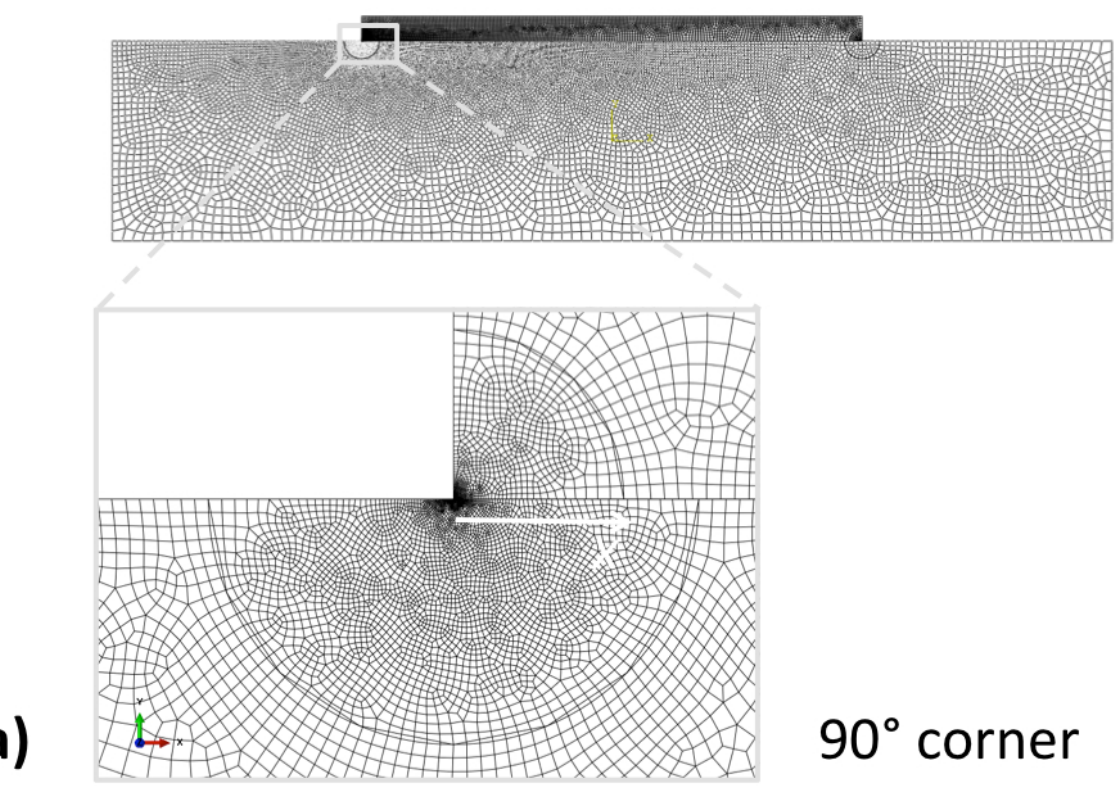

$90^{\circ}$ corner

b)

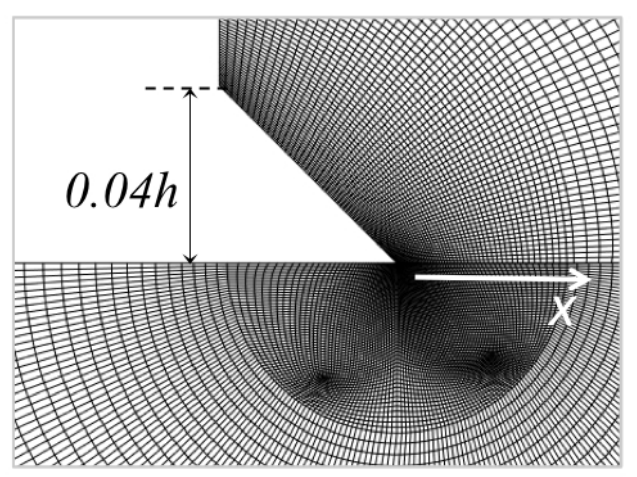

\section{$45^{\circ}$ corner}

c)

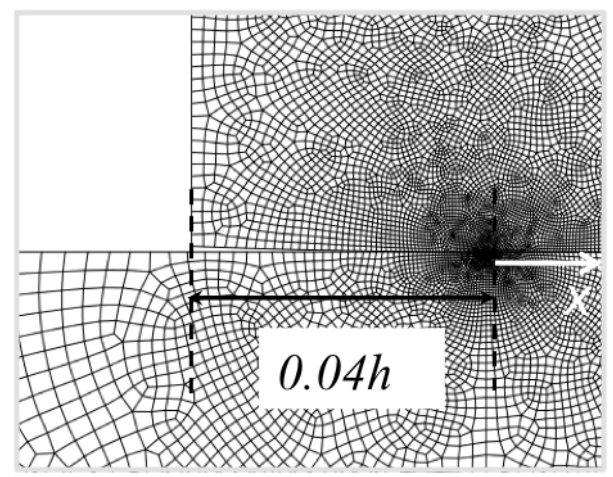

\section{smooth}

Figure 6: Three corners with the same macroscopic geometry as Fig. 2: (a) a $90^{\circ}$ external corner; (b) a $45^{\circ}$ external angle for a depth of $0.04 h$; (c) a smooth corner formed by an arc of a circle of radius $h$ meeting the interface at a tangent at a distance of $0.04 h$ from the edge. 
(a)

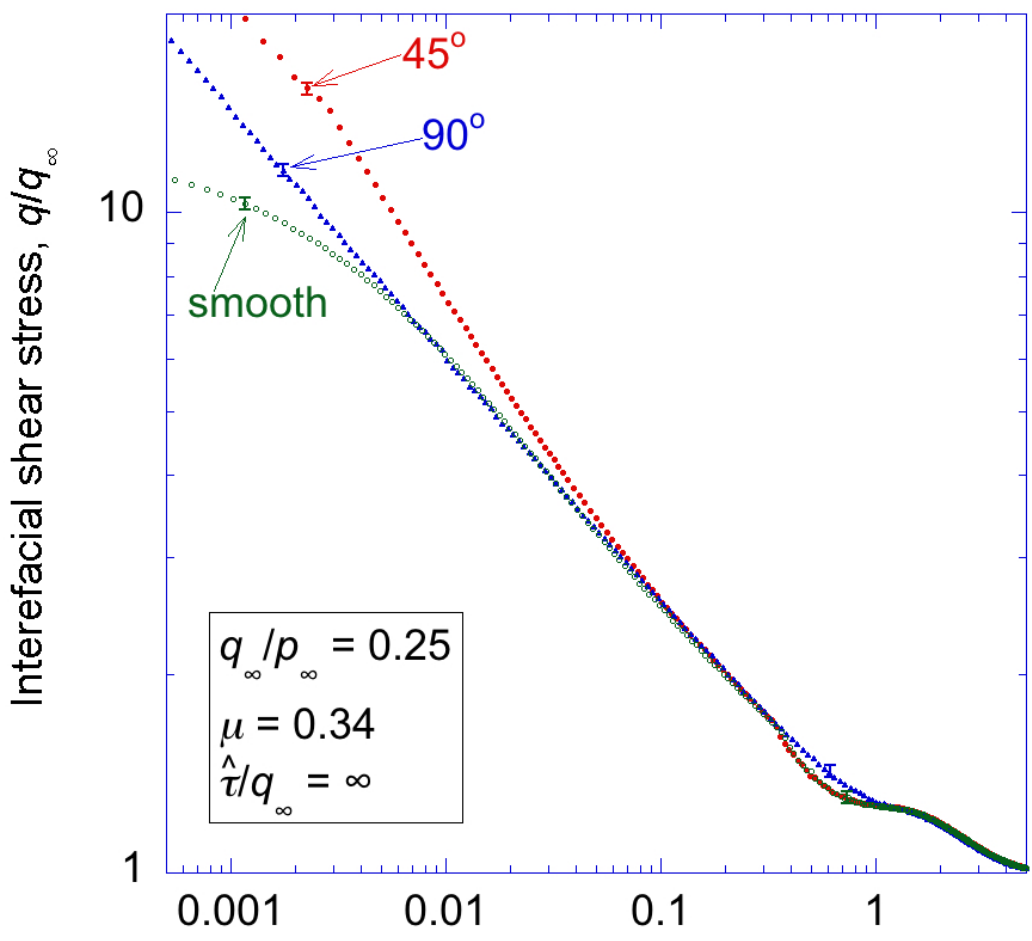

Distance from corner, $x / h$

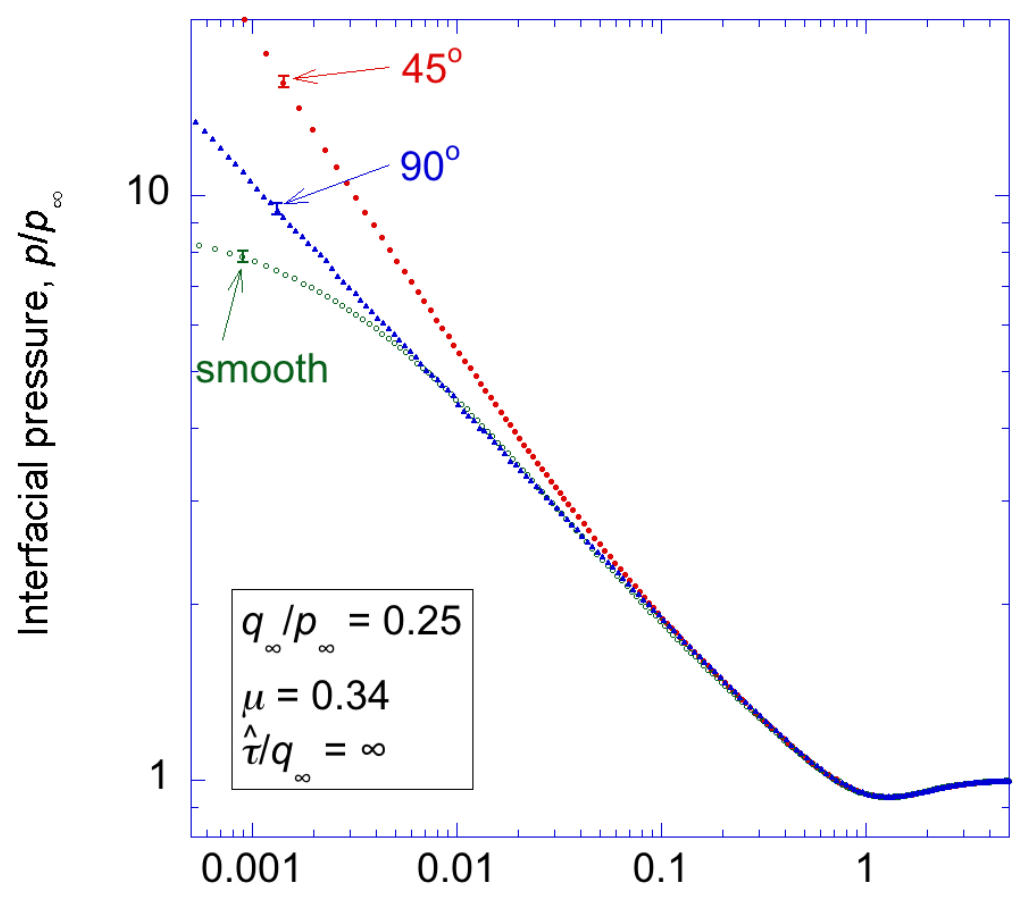

(b) Distance from corner, $x / h$ 
(c)

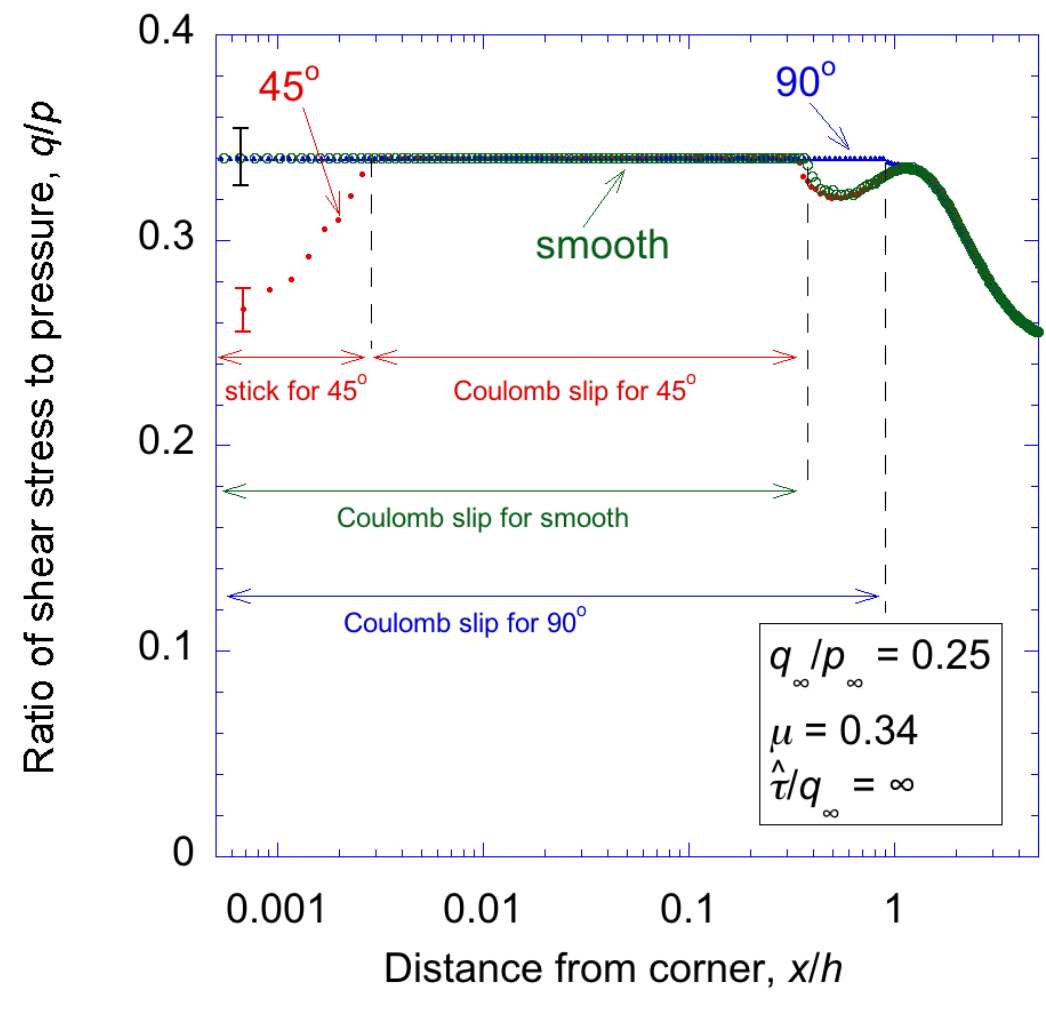

Figure 7: Plots of how the (a) shear stress, (b) normal pressure, and (c) ratio of shear stress to normal pressure vary along an interface for the three different corners of Fig. 6, with a coefficient of friction given by $\mu=0.34, q_{\infty} / p_{\infty}=0.25$, and an infinite shear strength.

The stresses for the same three geometries are shown in Fig. 8 for the case when there is a finite shear strength, assumed to be $\hat{\tau} / q_{\infty}=4.8$. This fixed value of $\hat{\tau} / q_{\infty}$ leads to slightly different values for the instantaneous cohesive-length scales for the three geometries: $\xi_{I I} / h=0.13,0.24$ and 0.21 for the $90^{\circ}, 45^{\circ}$ and smooth contacts, respectively. As can be seen from Fig. 8(c), the slip conditions are much less sensitive to the precise details of the contact than in the previous case. Furthermore, the total slip distances at the corners of these three geometries very by less than a factor of $50 \%$. When only Coulomb friction operates, there is an order of magnitude difference in the slip distances, even for the two cases where slip did occur at the corner. 
(a)

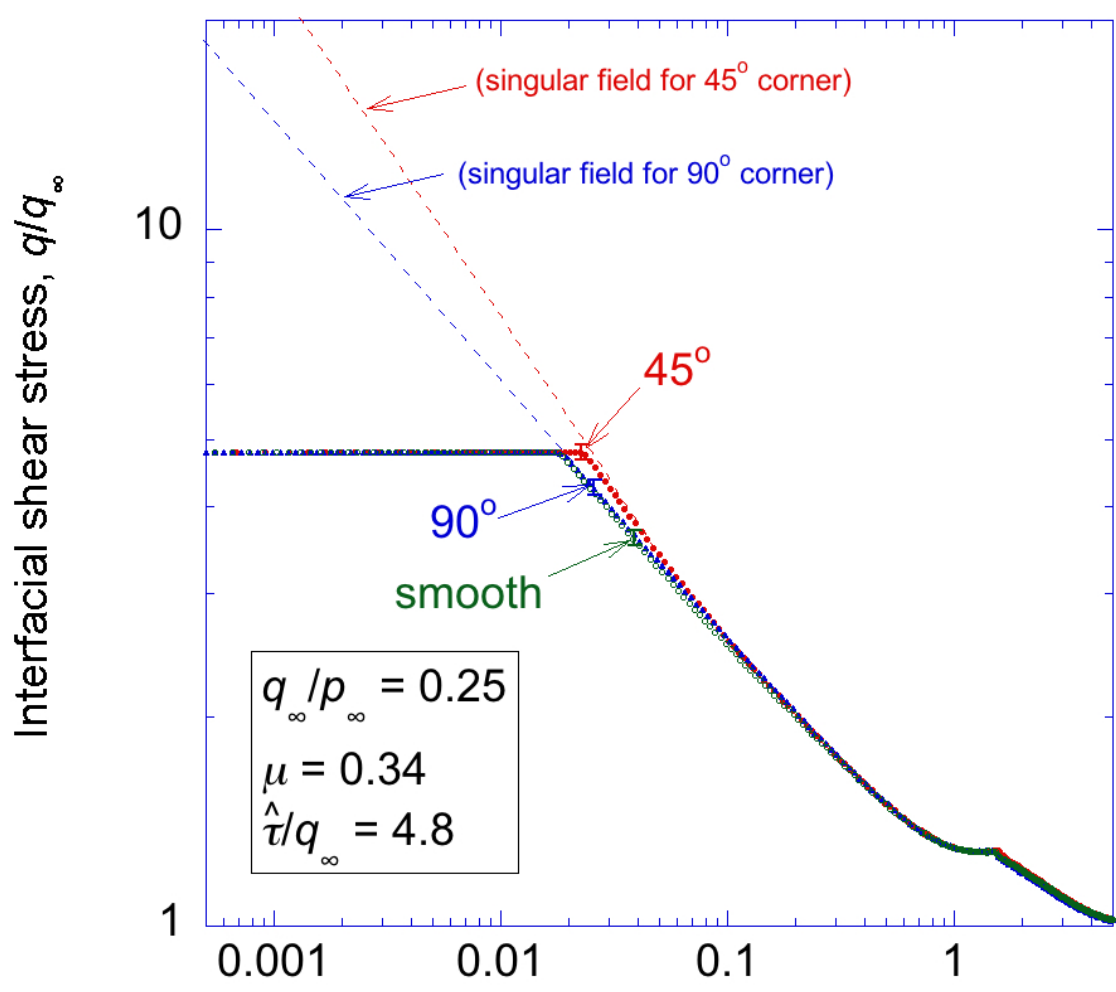

Distance from corner, $x / h$

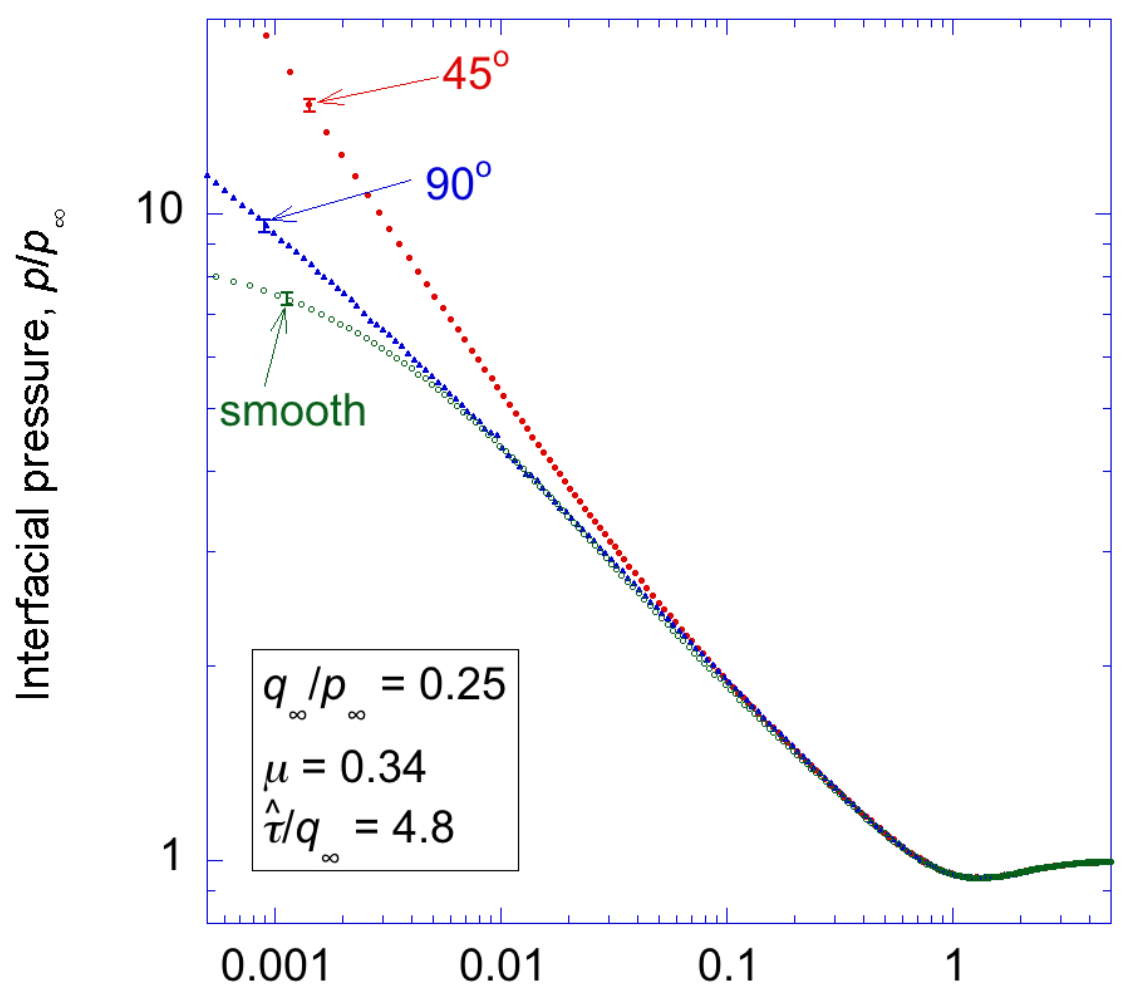

(b) 
(c)

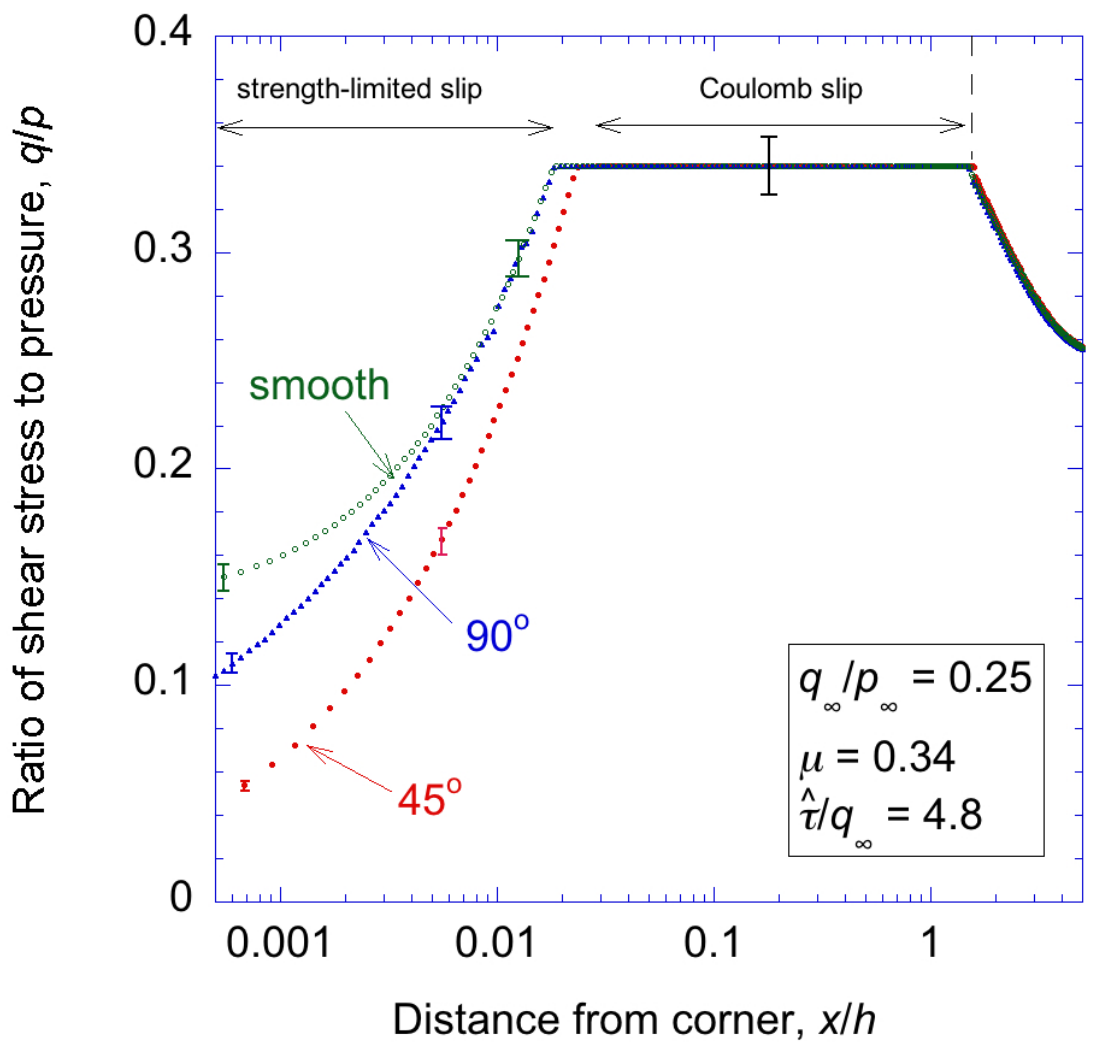

Figure 8: Plots of how the (a) shear stress, (b) normal pressure, and (c) ratio of shear stress to normal pressure vary along an interface for the three different corners of Fig. 6 , with a coefficient of friction given by $\mu=0.34, q_{\infty} / p_{\infty}=0.25$, and $\hat{\tau} / q_{\infty}=$ 4.8 .

\subsection{Effects of interfacial shear strength on wear propagation}

Partial slip can cause wear. This modifies the profile of the contact surfaces and redistributes the stress. Studies have shown that if Coulomb's law is assumed for friction, the stick-slip boundary does not move as wear progresses [24, 25]. This can be rationalized from a fracture-mechanics perspective. The local wear in the slip region evolves to a crack-like feature. The shear stresses and normal pressure will, therefore, have an inverse square-root dependence ahead of the wear scar, with the mode-I and mode-II stress-intensity factors being proportional to the applied shear and normal tractions. This means that $q / p=q_{\infty} / p_{\infty}$, so, if the conditions for macroscopic slip 
are not met $\left(\right.$ i.e., $q_{\infty} / p_{\infty}<\mu$ ), partial-slip in the singular region ahead of the wear scar will not be occur either. This is of practical importance, because it would imply that a wear scar cannot proceed beyond the initial stick-slip boundary. However, as will be shown in the calculations that follow, a finite shear strength always results in slip ahead of a crack-like feature, so a finite interface strength can result in the propagation of a wear scar across an interface.

To demonstrate this effect, we generated a worn contact interface and simulated a slip problem. As an example, we used a model with the dimensions and loading conditions of Fig. 2. The friction coefficient was set equal to 0.38 , and the shear strength was switched on or off by setting $\hat{\tau} / q_{\infty}$ equal to either 3 or $\infty$. These values were chosen to establish a relatively small initial slip zone. We then developed a wear scar by modeling wear according to Archard's law [26]:

$$
w=A p \delta
$$

where $A$ is the wear coefficient, and $\delta$ is the nodal slip. ${ }^{5}$ We analyzed one cycle of increasing $q_{\infty}$ from zero up to a maximum value, and back again. We computed the normal pressure and slip distance at each node in the contact region during each increment of a single cycle. We then used Eq. 11 to compute the integrated wear for each node, and then modified the mesh at the interface to create a wear scar, using the updating method of fictitious eigenstrains described in Ref. [27].

A wear scar typically forms during many loading cycles, with each cycle changing the morphology slightly. We ignored this aspect of the problem for the purposes of

\footnotetext{
${ }^{5}$ Archard's law might be better expressed in terms of $q$, not $p$, to account better for the frictional work done with a finite shear strength. However, this consideration doesn't matter here, since we focus on whether there is slip, or not, to determine whether there is wear.
} 
this study, and investigated the limit of the process by scaling the wear coefficient, $A$, so that contact was completely lost within the slip zone after just one cycle of loading. Using the new geometry with this computed wear scar at the interface (Fig. 9), we examined the stress state along the interface when $q_{\infty}$ was increased to its original maximum value.

The distributions of the shear stress, normal stress, and the corresponding ratios of the shear and normal stress are shown in Fig. 10. With an infinite shear strength, corresponding to Coulomb slip, the interfacial stresses exhibit a crack-like singularity (inverse root) for the stresses (not that the normal pressures also exhibit singular behavior, because the wear scar has some depth). The ratio of the stresses in the singular field scales with $q_{\infty} / p_{\infty}$, as might be expected from a fracture mechanics analysis of the crack problem. This means that if the coefficient of friction is high enough to prevent general slip along the interface, it will also prevent partial slip ahead of a wear scar. This is consistent with the analysis of Ref. [24], and may suggest a general result that the stick-slip boundary does not move in response to wear if only Coulomb friction is assumed.

However, when the calculations are repeated with a finite shear strength (with $\hat{\tau} / q_{\infty}=3$ ), slip occurs ahead of the wear scar. Again, this is expected by analogy to cohesive zones at crack tips. This extended zone of partial slip means that a wear scar can propagate across an interface. Its formation is not limited to the initial region of partial slip, as would be predicted from an assumption of only a Coulomb law. This provides an important example of why the introduction of finite shear strengths can have a significant role in the analysis of wear at the corners of contacts. 


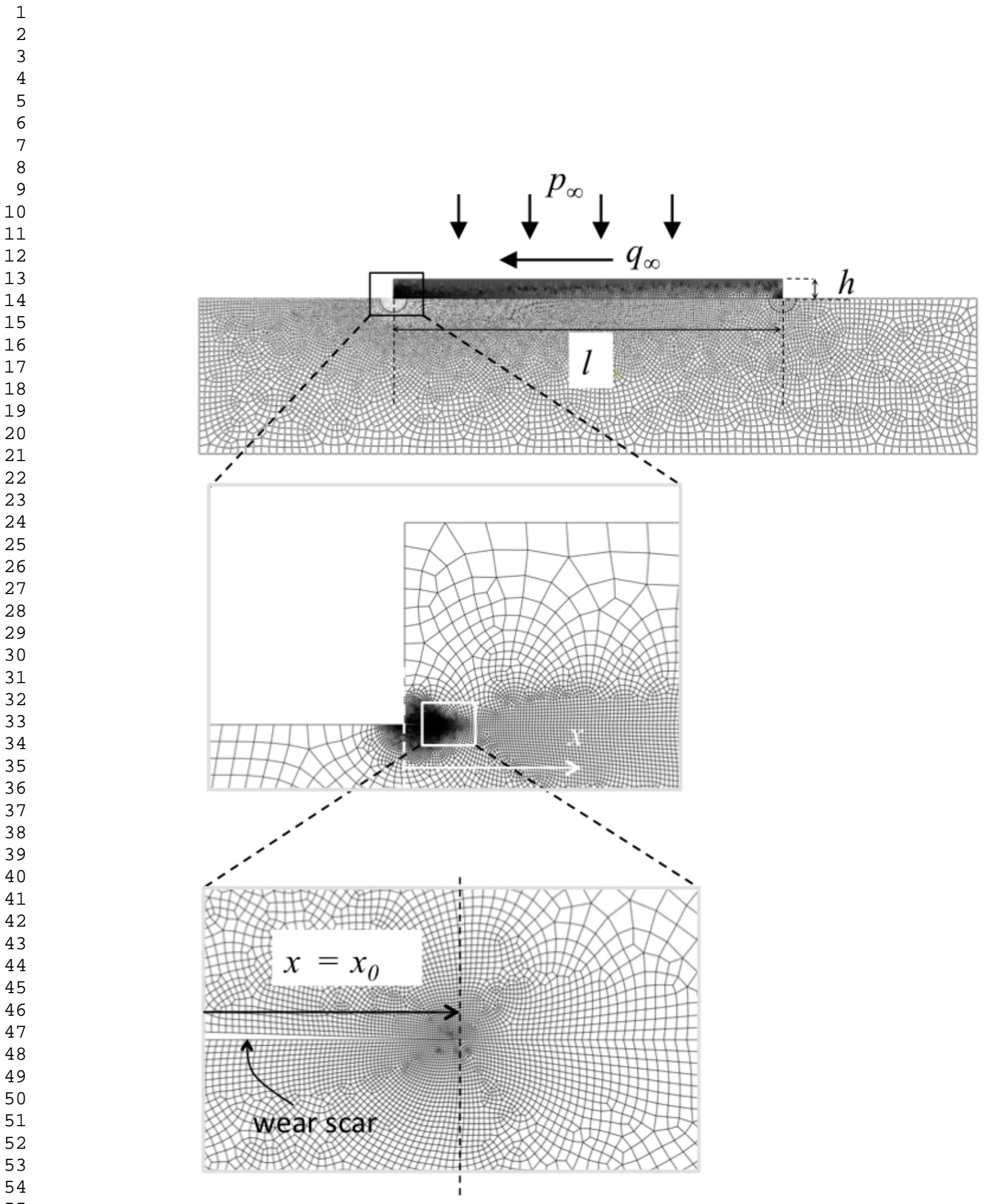

55

56

Figure 9: The wear scar is created by adjusting the nodal coordinates of the mesh on the basis of a wear calculation that results in all contact being lost within the initial slip zone, after a single cycle of slip. 
(a)

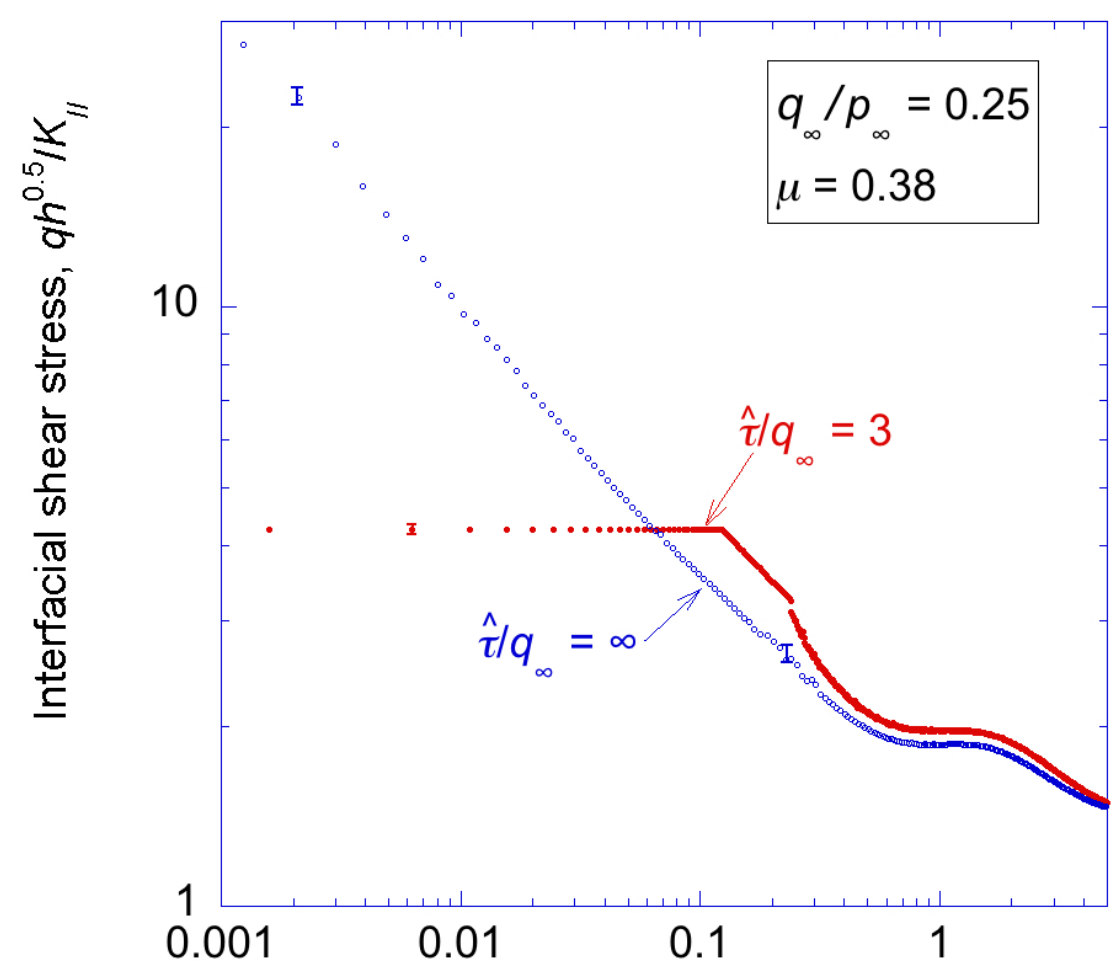

\section{Distance from tip of wear scar, $\left(x-x_{0}\right) / h$}

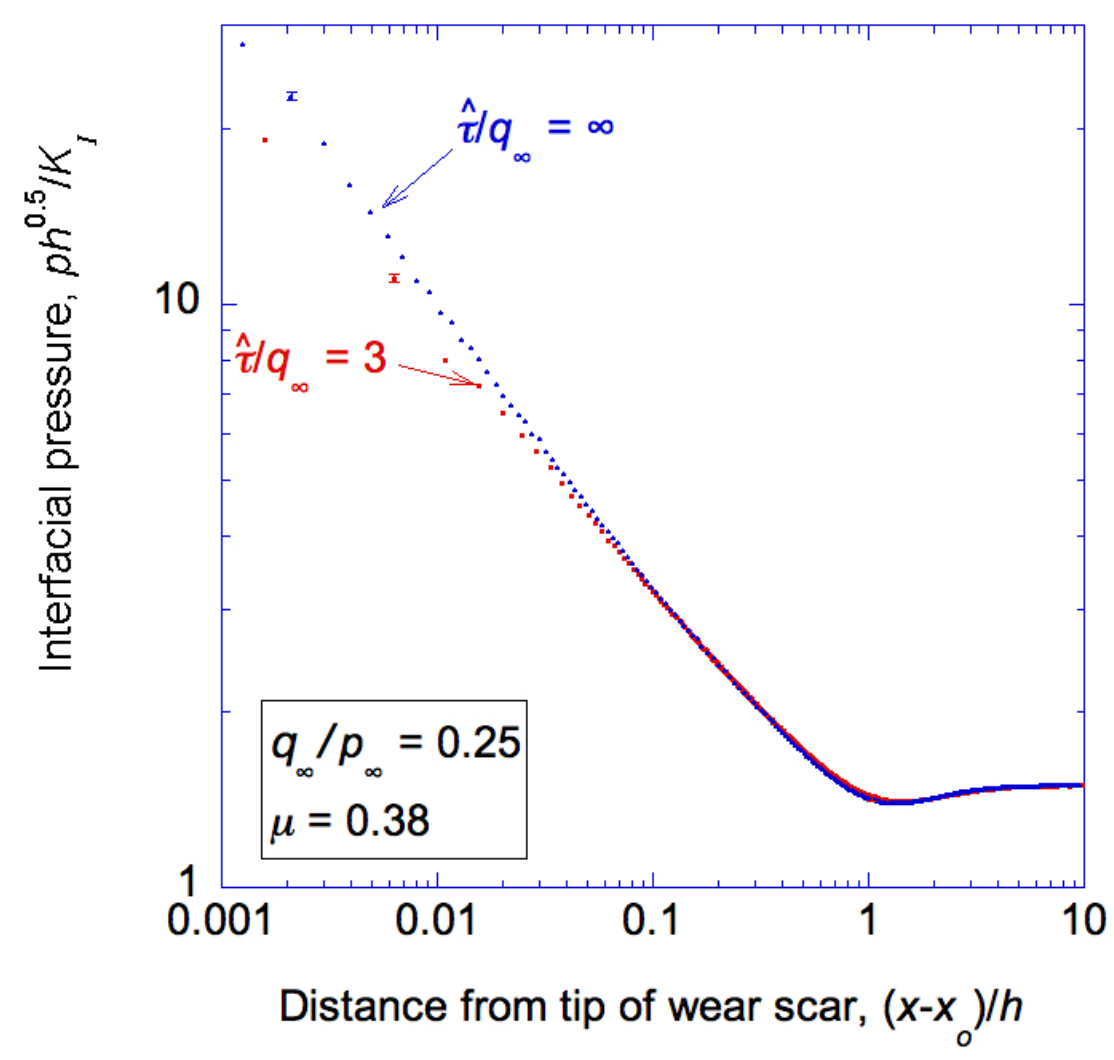

(b) 
(c)

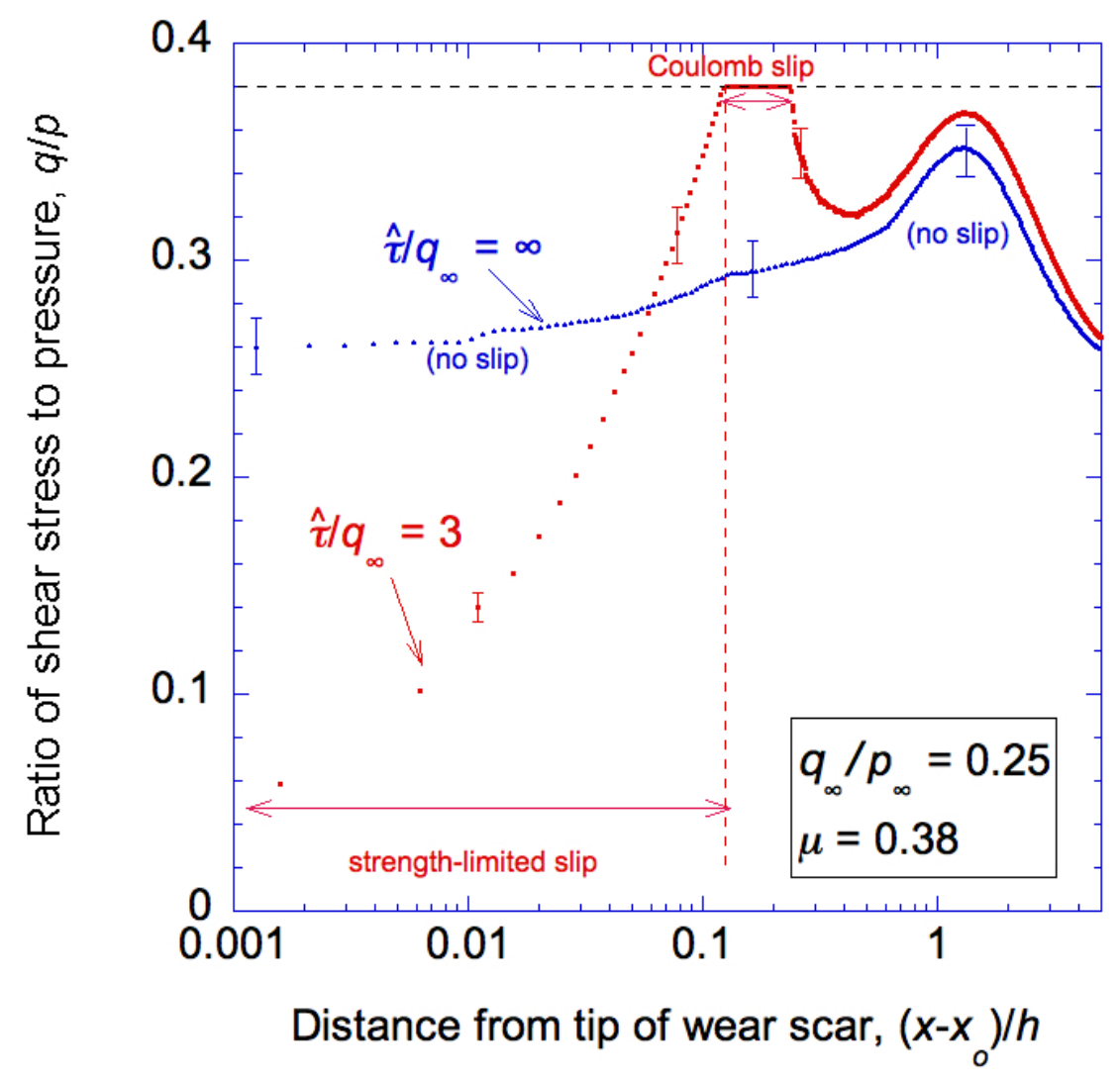

Figure 10: (a) Shear stress and (b) pressure distributions along the interface ahead of a wear scar with $\mu=0.38$, comparing the behavior of an interface that follows a pure Coulomb law with one that has a finite shear strength. The asymptotic stress fields correspond to those expected at the tip of an interfacial crack. (c) The corresponding distributions of the ratio between the shear stress and pressure. If only a singular Coulomb law is assumed for the interface, the ratio of the stresses at the crack tip is given by the ratio of $K_{I I} / K_{I}$, which is equal to $q_{\infty} / p_{\infty}$. Therefore, there is no partial slip ahead of the wear scar, and, hence, no propagation of the wear. Conversely, the assumption of finite shear strength, results in a slip zone being generated ahead of the wear scar. This will lead to further wear, and the propagation of wear across the contact. 


\section{Conclusions}

The integration of a finite interfacial strength with a Coulomb friction law has a significant influence on slip and wear at the corner of a contact. It is possible to define an instantaneous cohesive length in terms of the modulus, slip distance and interfacial strength. A comparison of this length with a characteristic geometrical scale gives an indication of when small-scale conditions are appropriate, and when the stress field approaching the corner can be reasonably approximated by the singular field. In particular, when the instantaneous cohesive-length scale, $\xi_{I I} / h$, is small, the stresses along the interface near the corner are uniquely described by

$$
\frac{q h^{1-\lambda}}{K}=f\left(\frac{x}{h}, \frac{\xi_{I I}}{h}, \mu\right),
$$

where $K$ and $\lambda$ are functions of $\mu$. For larger values of $\xi_{I I} / h$, the ratio of $q_{\infty} / p_{\infty}$ is no longer captured by the stress-intensity factor, so this becomes an additional non-dimensional group that affects the stresses.

Cohesive zones have an advantage of capturing the essence of elastic solutions where they are appropriate, while describing behavior in the regimes and length scales where elastic solutions are not valid. As with fracture, the use of cohesive-zone concepts reduces the sensitivity of the problem to local details of the singular stress field. Depending on the scale at which a corner is described, the singular fields and corresponding description of slip along the interface can be very different for geometries with similar macroscopic descriptions at a larger scale. The introduction of a cohesive length reduces this sensitivity.

If there is partial slip, the resulting wear will cause a crack-like geometry to evolve along the interface up to the slip-stick boundary. However, with the assumption of 
Coulomb friction, the wear scar will not evolve beyond the original partial-slip boundary, as has been established for the Hertz contact problem [28], in which the normal stresses are not singular, but the shear stresses are [24]. The introduction of a finite interfacial strength results in the slip-stick boundary moving with the propagation of wear. This is expected to be of significance to models of wear, since it allows a wear scar to propagate across a macroscopically non-slipping contact.

\section{Acknowledgements}

We would like to acknowledge and thank Prof. J. R. Barber for very valuable discussions. ZH thanks Mr. H. Wang for useful discussions, and for contributing to the calculations used to investigate mesh sensitivity. This research was supported by the Consortium for Advanced Simulation of Light Water Reactors (http://www.casl.gov), an Energy Innovation Hub (http://www.energy.gov/hubs) for Modeling and Simulation of Nuclear Reactors under U.S. Department of Energy Contract No. DE-AC0500OR22725. 


\section{References}

[1] D. W. Hoeppner. Fretting fatigue case studies of engineering components. Tribology International, 39:1271-1276, 2006.

[2] K. L. Johnson. Contact Mechanics. Cambridge University Press, Cambridge, UK, 1984.

[3] R. B. Sills and M. D. Thouless. Cohesive-length scales of intrinsic and extrinsic toughening mechanisms in composites. International Journal of Solids and Structures, 55:32-43, 2015.

[4] J. P. Parmigiani and M. D. Thouless. The effects of cohesive strength and toughness on mixed-mode delamination of beam-like geometries. Engineering Fracture Mechanics, 74:2675-2699, 2007.

[5] R. B. Sills and M. D. Thouless. The effect of cohesive-law parameters on mixedmode fracture. Engineering Fracture Mechanics, 109:353-368, 2013.

[6] O. Vingsbo and S. Söderberg. On fretting maps. Wear, 126:131-147, 1988.

[7] Z. R. Zhou, K. Nakazawa, M. H. Zhu, N. Maruyama, P. Kapsa and L. Vincent. Progress in fretting maps. Tribology International, 39:1068-1073, 2006.

[8] N. Bay and T. Wanheim. Real area of contact and friction stress at high pressure sliding contact. Wear, 38:201-209, 1976.

[9] M. Ödfalk and O. Vingsbo. An elastic-plastic model for fretting contact. Wear, 157:435-444, 1992.

[10] L. Chang and H. Zhang. A mathematical model for frictional elastic-plastic sphere-on-flat contacts at sliding incipient. Journal of Applied Mechanics, 74:100-106, 2007.

[11] P. D. Warren, T. J. Mackin and A. G. Evans. Design, analysis and application of an improved push-through test for the measurement of interface properties in composites. Acta Metallurgica et Materialia, 40:1243-1249, 1992.

[12] D. B. Marshall, M. C. Shaw and W. L. Morris. Measurement of interfacial debonding and sliding resistance in fiber reinforced intermetallics. Acta Metallurgica et Materialia, 40:443-454, 1992.

[13] M. D. Thouless, J. W. Hutchinson and E. G. Liniger. Plane-strain, bucklingdriven delamination of thin-films - Model experiments and mode-II fracture. Acta Metallurgica at Materialia, 40:2639-2649, 1992. 
[14] D. B. Bogy. Two edge-bonded elastic wedges of different materials and wedge angles under surface tractions. Journal of Applied Mechanics, 93:377-386, 1971.

[15] D. A. Hills and D. Dini. Characteristics of the process zone at sharp notch roots. International Journal of Solids and Structures, 48:2177-2183, 2011.

[16] R. Flicek, D. A. Hills and D. Dini. Progress in the application of notch asymptotics to the understanding of complete contacts subject to fretting fatigue. $F a$ tigue and Fracture of Engineering Materials and Structures, 36:56-64, 2013.

[17] J. R. Barber. Elasticity. Springer, Dordrecht, Netherlands, 2010.

[18] C. M. Churchman and D. A. Hills. General results for complete contacts subject to oscillatory shear. Journal of the Mechanics and Physics of Solids, 54:1186$1205,2006$.

[19] E. E. Goutos and P. S. Theocaris. Stress concentrations at the apex of a plane indenter acting on an elastic half plane. Journal of Applied Mechanics, 42:688$692,1975$.

[20] M. Comninou. Stress singularity at a sharp edge in contact problems with friction. Journal of Applied Mathematics and Physics (ZAMP), 27:493-499, 1976.

[21] A. Hillerborg, M. Modéer and P. E. Petersson. Stress concentrations at the apex of a plane indenter acting on an elastic half plane. Journal of Applied Mechanics, 42:688-692, 1975.

[22] G. Bao and Z. Suo. Remarks on crack-bridging concepts. Applied Mechanics Review, 45:355-366, 1992.

[23] SIMULIA Dassault Systèmes. Abaqus Analysis User's Manual. http://www.maths.cam.ac.uk/computing/software/abaqus_docs/docs/v6.12/ books/usb/pt09.html. Accessed on May 25, 2015.

[24] I. G. Goryacheva, P. T. Rajeev and T. N. Farris. Wear in partial slip contact. Journal of Tribology, 123:848-856, 2001.

[25] J. Ding, S. B. Leen and I. R. McColl. The effect of slip regime on fretting wearinduced stress evolution. International Journal of Fatigue, 26:521-531, 2004.

[26] J. F. Archard. Contact and rubbing of flat surfaces. Journal of Applied Physics, 24:981-988, 1953.

[27] Z. Hu, W. Lu, M. D. Thouless and J. R. Barber. Simulation of wear evolution using fictitious eigenstrains. Tribology International, A82:191-194, 2015. 
[28] H. Hertz. Ueber die Berührung fester elasticher Körper. Journal für die reine und angewandte, 92:156-171, 1882. (As translated by D. E. Jones and G. A. Schott in "Miscellenaneous Papers" by H. Hertz, Macmillan \& Co., New York, NY, USA, 1896). 


\section{Graphical abstract}

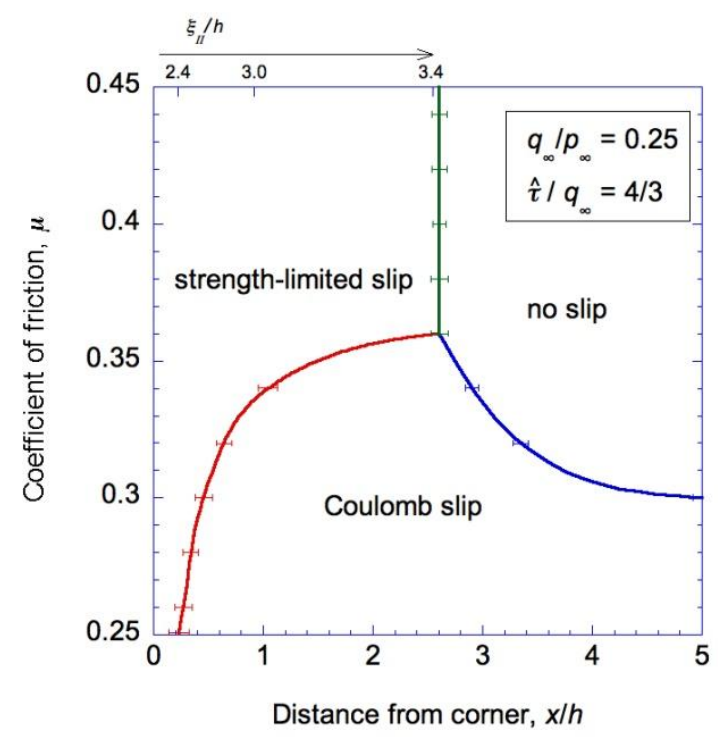

